\title{
Simulated-Person Methodology Workshop: Enhancing Pedagogical Practices within Higher Education
}

\author{
Eva Hava Peisachovich, Samantha Johnson, Iris Epstein, Celina Da Silva, Raya Gal, Lora \\ Appel, Celia Popovic \\ York University, Toronto, Canada
}

\begin{abstract}
Interaction between organizations, tasks, and individual providers influences human behaviour, affects organizations' or systems' performance, and is a key component of professional-competence development. Further, ineffective communication between professionals is deemed a contributing factor to adverse events. This underscores the need to provide educators with the tools and education to embed methods in their teaching that will enable learners to effectively intervene in highly charged interpersonal situations and high-risk scenarios; these concerns highlight the value of realistic simulated-experiential approaches, such as the one proposed in this project. The first phase of this project involved an experiential workshop, developed and conducted at a Canadian university; the workshop was designed to provide educators with knowledge and skills to work with and effectively utilize simulators, enhancing pedagogical classroom practices for teaching undergraduate learners. The workshop provided educators with opportunities for meaningful reflection on their teaching practice and the ability to apply this insight to optimize student learning. It provided theatre students, recruited as simulators as part of this interdisciplinary initiative, to expand their experiences and this will lead to an expanded practicum course for their program. This paper reflects on the workshop experiences and feedback obtained to provide an understanding of the participants' experiences.
\end{abstract}

\section{Introduction}

While much research exists on the impact of simulated-person (SP) methodology on students' skills acquisition, there is little scholarly literature that discusses how best to educate both educators and actors/simulators on how to effectively use this methodology. The aim of this project is to provide educators with the tools needed to apply and embed SP methodology in their teaching and to work with simulators in the context of a teaching-learning setting. In addition, the project proposes a potentially sustainable and affordable model for the training and delivery of SP methodology. To meet this aim, in the summer of 2016 we conducted a workshop as part of an innovative initiative to embed SP methodology within one Canadian university and gathered data from the participants regarding its efficacy; this workshop was piloted at the university's School of Nursing. We hypothesized that the workshop would aid educators in gaining a deeper understanding of SP methodology, its use and benefits, and of strategies to effectively embed this methodology within their teaching. Additionally, an overall goal of this project was to incorporate sustainability and extend the model into a universitywide initiative. A second iteration, which built on the promising results of the 2016 SP methodology workshop, was offered to multiple disciplines at the university. Selected highlights from the most recent workshop, which was held in June 2017, will be discussed to demonstrate the growth and progress of this innovative project.

There is little research available that discusses this unique aim and evaluates the process of providing educators with the knowledge and skills to embed this pedagogical methodology in their classrooms. The databases CINAHL: Cumulative Index to Nursing and Allied Health (EBSCO) and Nursing and Allied Health Source (ProQuest) were utilized to conduct a search of available literature. The search terms simulation and education were used to conduct the initial search and other articles were sourced from the reference lists of the populated results. Abstracts were reviewed, and papers that did not relate to simulation in the context of education were removed. The articles selected for this review ranged from the areas of nursing education and medical education to political-science education and other interprofessional disciplines. We learned that while the research indicates that simulation is an effective teaching tool, it can be expensive [1] and thus 
remains underutilized as a pedagogical tool in undergraduate education beyond medicine and nursing.

Given this, it is important to explore both how to support educators so they may effectively use SPs and how to embed this methodology in a cost-effective manner. Our proposal, which prepares and includes theatre students as simulators through the creation of a practicum course in simulation, is one such method and the workshop upon which the project is based allowed us to lay the foundation for such an initiative at a Canadian University.

\section{Relevant scholarship}

Simulation is a teaching strategy based in experiential learning; it facilitates activities that mimic real-life situations [2] [3]. The learner is asked to perform certain skills in the simulation context in order to engage in learning or to determine preparedness for a situation [4]. There is a large research focus on clinicalskill acquisition as a learning objective of simulation across the health professions. However, simulation activities can also be utilized for many other applications, including the development of competence in communication, professionalism, cultural sensitivity, and ethics; these applications receive less research emphasis [5], particularly in professions outside of health care.

The benefits of simulation include increased confidence in practice, new knowledge generation, and skill acquisition [2] [6]. This learning strategy supports a safe educational environment in which one can practice and make mistakes without risk to actual persons or in actual environments [7]. The use of simulation-based methodology should involve premeditation of how this learning experience will enhance theory-praxis understanding [8]; this was a starting point for the project. The process of developing simulation scenarios requires thoughtful engagement, informed by sound pedagogical frameworks, to support robust learning opportunities [9] [10]. It is also important to evaluate the level of authenticity required in order for the simulation to engage learning and suspend disbelief [8]; this concern should be addressed during the planning process. To ensure that these important elements of simulation-based education were honoured in our project, we chose to locate our 2016 pilot program in the department of nursing as these faculty members were already somewhat familiar with simulation pedagogy and the nature of authentic casebased learning.

While there is a wealth of literature supporting simulation as a validated tool for assessment and learning, many authors agree that there is a lack of empirical evidence related to how scenario- development guidelines and evaluation tools for outcome measurement [3] [6] [8] are related to the effectiveness of simulation. Anecdotal evidence and studies that largely evaluate perceptions rather than empirical data contribute to unanswered questions regarding the application of simulation [4] [7]. Research regarding the specific scope of this project is scant and testifies to the value that this information will contribute to the body of literature regarding simulation as an educational methodology in undergraduate education. Given that knowledge and skills within the context of simulation-based pedagogy_particularly the use and application of simulated-person methodology - may be limited to educators in health and aviation, this workshop provided further insight into the need for collaborative knowledge and understanding to embed this methodology in the classroom in the broader university setting.

\section{Methods}

This pilot workshop was guided by the appreciative-inquiry framework, a strengths-based approach that operates under the basic assumption that "every living system has rich and inspiring accounts of the positive, suggesting that in every organization, group or society something "works"' [11] (p. 260). Appreciative inquiry focuses on successes and achievements, guiding participants to magnify and proliferate these positive attributes [11]. This framework was developed to initiate and manage personal and organizational change through identification of strengths [12]. As a group of researchers and educators with a breadth of experience in simulation methodology, we chose appreciative inquiry as a method that would allow us both to investigate the strengths of simulation and simulatedperson methodology as an experiential tool and to conceptualize a design that could be applied in a multidisciplinary environment. This model of appreciative inquiry was threaded through the second iteration of the simulated-person methodology workshop held on June 28-29, 2017.

Appreciative inquiry (AI) can be used in multiple ways through research methods that empower participants [12]. Its use supports the fundamental learning methods of inquiry, dialogue, reflection, collaboration, and feedback [13]. In nursing practice, there often exist underlying anxieties that can affect group dynamics; these anxieties can be better understood utilizing an AI framework to highlight strengths while confronting damaging group dynamics [14]. The AI framework supports the creation of a safe environment that promotes personal sharing and the development of deep connections through respect for 
uniqueness and acknowledgement of diversity [12]. Further, the framework aligns with the exploratory nature of this project to inform teaching and learning through the application of simulated-person methodology. AI was, therefore, included in the design of the workshop. The workshop utilized a collaborative model in which simulators and educators were trained in the pedagogy together. Imbedding $\mathrm{AI}$ in the design of the workshop was intended to

- enhance participants' understanding of their roles in the process;

- $\quad$ lead to the development of rich and authentic scenarios, as nurse educators provided content and simulators worked with them to make the content play out authentically;

- $\quad$ promote the exploration of possible best practices;

- $\quad$ promote reflection and feedback on each module of the workshop in order to inform future iterations of the workshop;

- develop relationships between educators and simulators and, thus, create a safe environment for sharing and deep reflection;

- investigate the strengths of human simulation in the translation of theory to practice; and

- maximize learning for participants and, therefore, for students.

A descriptive mixed-method design was employed to gain a better understanding of the participants' experiences of the workshop and their ability to use, apply, and engage with SP methodology within a teaching-learning context. Qualitative data collection was used to determine whether the workshop increased the knowledge and skills of participants (faculty and simulators). Quantitative data collection was used to gain an understanding of whether and how the organization and design of the workshop met the participants' learning needs and facilitated their ability to sustain the knowledge and skills learned.

\subsection{Participants}

For the June 2017 SP methodology workshop, the methods for sampling were kept consistent, but the modes of communication for recruiting new simulators were expanded. The workshop was advertised and registration was conducted through a website (http://spm.info.yorku.ca/) specifically created as part of the larger project to mobilize knowledge related to SP methodology. The staff members were affiliated with various departments, including the Teaching Commons, Health, Glendon Campus, the Department of Community Safety and the School of Kinesiology and Health Science. This diversified interest attests to the versatility of this methodology in endless environments. The workshop was conducted at York University and included six faculty members from various fields and six simulators recruited from the university's theatre program.

\subsection{Workshop design}

The aim of the 2017 workshop was to increase faculty participants' knowledge, skills, and confidence to engage and collaboratively work with simulators and to embed SP methodology in teaching-learning contexts. The workshop was grounded by rolemodelling strategies to promote an emotionally and physically safe container or environment [15].

As previously discussed, the June 2017 workshop built on the promising results of a three-day, intensive educational workshop that was held in July and August 2016 and piloted at the School of Nursing, prior to the term in which this methodology would be applied. Findings from the first workshop study were incorporated into the design of the 2017 workshop; for example, based on feedback, the workshop was distilled into two days, while maintaining the highly interactive nature of the pilot. This second iteration held to the program goals of providing foundational, practical information about simulated-person methodology to enhance educational experiences for learners. There was ample opportunity provided for application of this pedagogy and collaboration with simulators to actualize and fine-tune the scenario development process.

Learning methods included introduction to SP methodology for both small and large groups. Participants each received a binder containing reading materials, including information forms and copies of journal articles detailing studies used to embed SP methodology. The binder also provided each participant with a copy of the workshop's PowerPoint presentations, a resource list for teaching and learning with simulation, an overview of the advocacy-inquiry method of debriefing, a sample informed-consent form, a template for scenario writing, reflection activities, strategies for creating a safe space, and a dictionary of simulation-methodology terms and concepts. Throughout the day and at the end of each module, participants could record reflections, questions, ideas, and feedback.

Day 1 of the two-day workshop began with an overview of the agenda and discussion of activities planned for each of the days. Participants introduced themselves and shared personal experiences with SP methodology. This was followed by an engaging presentation on the components of simulation-based and experiential learning. The afternoon session saw participants interacting with the concepts of simulation and role play. Considerable time was spent looking at 
the main differences between simulation and role play before providing hands-on experience with the scenario-development process. It was stressed to participants that, while simulation is a form of role play, simulators are often highly trained in their respective roles, while role play is impromptu and often happens among peers. Simulators are aware of the learning objectives and have been trained to prompt thinking, guide scenarios, and respond to behaviours and affect in specific ways through shifts in their own affect or delivery of the scripted lines.

Day 2 built on the foundational aspects of the previous session and focused on learning the key components of scenario writing. Participants were encouraged to focus on learning outcomes as a guiding factor in the scenario-writing process. They became familiar with the kind of information needed to populate a scenario template both from an educational perspective and from the perspective of the simulators who would play the roles. The use of scripted lines and prompts to guide and redirect scenarios was also discussed. Workshop facilitators were readily available to provide feedback and clarification as needed. The afternoon session was highly interactive; roles were practiced, with educator-participants in the position of the learner experiencing simulation. The facilitators guided this experiential component by highlighting approaches appropriate with different types of learners and discussing the facilitation skills and the level of detail necessary to support SPs in simulation. The day's debriefing provided an opportunity to identify impressions, reflections, and outstanding learning needs.

Participants had an opportunity to gain experience in various roles in the simulation-based learning experience as they rotated from learner to facilitator, trained SPs in developed scenarios, interacted with a variety of learners, and iteratively fine-tuned written scenarios.
3.2.1. Sustainability of the content and engagement of participants in discussions. In total, four hours were spent on large-group activities and ten hours on smallgroup activities. The large-group sessions used didactic and interactive activities that covered content associated with SP methodology. The small-group sessions involved interaction between faculty, facilitators, and simulators. For example, a group of three individualsone faculty member, one facilitator, and one simulator-would work on a scenario, exchanging thoughts and ideas.

The roles of learner, educator, and simulator were interchanged to provide participants with a deeper understanding of how simulation works from different perspectives. Educators identified specific learner behaviours and were trained to portray these behaviours as a means of field testing the roles to ensure that they would be adjustable to individual learners. One facilitator and one SP worked at each station, while faculty members moved around as learners. Faculty/staff participants worked to develop scenarios and facilitate interactions with diverse learners, allowing them to observe how the scenarios were actualized as a teaching tool in practice. The objectives of this activity were to (a) allow faculty an opportunity to understand what it is like to be a learner, (b) provide SPs with experience in how roles can play out differently with different learners, and (c) provide faculty and SPs with deeper understanding of the facilitation process.

\subsection{Measures}

Given that the expectation of the workshop was to gain knowledge and skills to understand how to apply SP methodology in the classroom to meet objectives of a lesson, at the end of each day, a questionnaire (see Figure 1) was completed by the participants.

\footnotetext{
Questionnaire

1. Please describe the main ideas learned over the course of the workshop.

2. Describe any questions you have after completing this workshop.

3. Provide one way you would apply this to your teaching.

4. What would I tell a friend or colleague about this workshop?

5 . What areas were unclear or require further clarification?

6. Please share any suggestions for future workshops.

7. What other topics, tools would I need further training or support with?
}

Figure 1. Qualitative Data Collection Questions-SPM Workshop 2017

The questionnaires gathered feedback on the process and learning that occurred during the workshop. Additionally, on the final day of the workshop, participants completed a survey designed to assess the effectiveness of workshop organization and delivery (see Table 1). 


\subsection{Data analysis}

The quantitative data was analyzed using descriptive statistics. The analysis included each participant's total score on the organization and delivery of the workshop. Quantitative and qualitative results were merged during interpretation. Themes identified during quantitative data analysis were compared with qualitative results to explain and describe findings about the workshop and knowledge obtained. The qualitative data gathered over the course of the SP methodology workshop was beneficial in articulating the experience of participants with this pedagogical framework. The feedback received was overwhelmingly positive with $100 \%$ of participants rating the workshop program very good or excellent. Additionally, $75 \%$ of participants indicated the medium of groups as excellent, and $62.5 \%$ felt the workshop was excellent in terms of advancing expertise in SP methodology (See Table 1). Fifty per cent of participants rated the workshop as good in helping develop confidence to implement SP methodology in their teaching.

Table 1. Effectiveness of workshop organization and delivery

\begin{tabular}{|c|c|c|c|c|c|}
\hline \multirow{2}{*}{$\begin{array}{l}\text { Rating Categories } \\
\text { How would I rate the following }\end{array}$} & \multicolumn{5}{|c|}{ Percentage of Respondents (\%) } \\
\hline & Poor Fair & Good & $\begin{array}{l}\text { Very } \\
\text { Good }\end{array}$ & Excellent & $\begin{array}{l}\text { No } \\
\text { Response }\end{array}$ \\
\hline 1. Workshop program & & & 50 & 50 & \\
\hline 2. Material provided & & & 37.5 & 62.5 & \\
\hline $\begin{array}{l}\text { 3. Balance between presentations, discussions, } \\
\text { and activities }\end{array}$ & & & 37.5 & 62.5 & \\
\hline 4. Time distribution of the workshop & & 12.5 & 37.5 & 50 & \\
\hline 5. Organization of the workshop & & & 50 & 50 & \\
\hline $\begin{array}{l}\text { 6. Group work was an effective medium during } \\
\text { the workshop }\end{array}$ & & 12.5 & 12.5 & 75 & \\
\hline 7. The workshop advanced my expertise in using & & & & & \\
\hline SP methodology & & & 25 & 50 & 25 \\
\hline $\begin{array}{l}\text { 8. The workshop advanced my expertise in } \\
\text { planning and dealing with SP methodology }\end{array}$ & & 12.5 & 12.5 & 62.5 & 12.5 \\
\hline $\begin{array}{l}\text { 9. The workshop increased my confidence } \\
\text { in implementing SP methodology in my teaching }\end{array}$ & & & 37.5 & 37.5 & 25 \\
\hline $\begin{array}{l}\text { 10. The feedback received over the course } \\
\text { of the workshop was valuable }\end{array}$ & & & 12.5 & 87.5 & \\
\hline $\begin{array}{l}\text { 11. The workshop provided opportunities to } \\
\text { engage with student colleagues from other } \\
\text { disciplines }\end{array}$ & & & 25 & 75 & \\
\hline $\begin{array}{l}\text { 12. Has the workshop provided me with the } \\
\text { necessary tools to develop and implement } \\
\text { scenarios for my course }\end{array}$ & & & 50 & 37.5 & 12.5 \\
\hline
\end{tabular}

The data from the 2017 workshop indicated the benefits of this instruction and interactive learning environment in building capacity to apply SP methodology in various settings. The quantitative data was analyzed using Statistical Package for the Social Sciences (SPSS) software. There were significant, positive correlations between the distribution of time of the workshop and the effectiveness of group work as a medium. Also, positive correlations were found between the participants' rating of the workshop's effectiveness in teaching how to create simulations that meet learning objectives and the indicators of increased confidence and expertise in planning, creating, and facilitating SP methodology (see Tables $2 \mathrm{a}$ and $2 \mathrm{~b}$ ). 
Table 2a. Correlations from SPSS Analysis for SP methodology workshop 2017

\begin{tabular}{|c|c|c|c|c|c|c|c|}
\hline & \multicolumn{6}{|c|}{ Question number } \\
\hline & & Q1 & Q2 & Q3 & Q4 & Q5 & Q6 \\
\hline \multirow[t]{2}{*}{ Q1. Workshop Program } & PC & 1 & .258 & -.258 & .539 & $1.000^{* *}$ & .539 \\
\hline & S2T & & .537 & .537 & .168 & .000 & .168 \\
\hline \multirow[t]{2}{*}{ Q2. Material Provided } & $\mathrm{PC}$ & .258 & 1 & .467 & $.788^{*}$ & .258 & .696 \\
\hline & S2T & .537 & & .244 & .020 & .537 & .055 \\
\hline \multirow{2}{*}{$\begin{array}{l}\text { Q3. Balance between } \\
\text { presentations, discussions } \\
\text { and activities }\end{array}$} & PC & -.258 & .467 & 1 & .417 & -.258 & .325 \\
\hline & S2T & .537 & .244 & & .304 & .537 & .433 \\
\hline \multirow{2}{*}{$\begin{array}{l}\text { Q4. Time distribution of the } \\
\text { workshop }\end{array}$} & PC & .539 & $.788^{*}$ & .417 & 1 & .539 & $.806^{*}$ \\
\hline & S2T & .168 & .020 & .304 & & .168 & .016 \\
\hline \multirow{2}{*}{$\begin{array}{l}\text { Q5. Organization of } \\
\text { workshop }\end{array}$} & PC & $1.000^{* *}$ & .258 & -.258 & .539 & 1 & .539 \\
\hline & S2T & .000 & .537 & .537 & .168 & & .168 \\
\hline \multirow{2}{*}{$\begin{array}{l}\text { Q6. Group/dyad work was } \\
\text { an effective medium during } \\
\text { the workshop }\end{array}$} & $\mathrm{PC}$ & .539 & .696 & .325 & $.806^{*}$ & .539 & 1 \\
\hline & S2T & .168 & .055 & .433 & .016 & .168 & \\
\hline \multirow{2}{*}{$\begin{array}{l}\text { Q7. The workshop taught } \\
\text { me to create roles for } \\
\text { simulation that meet } \\
\text { learning objectives for my } \\
\text { students }\end{array}$} & $\mathrm{PC}$ & .000 & .188 & .188 & -.044 & .000 & -.218 \\
\hline & S2T & 1.000 & .656 & .656 & .918 & 1.000 & .604 \\
\hline \multirow{2}{*}{$\begin{array}{l}\text { Q8. The workshop has } \\
\text { advanced my expertise in } \\
\text { planning, creating and } \\
\text { facilitating SPM }\end{array}$} & $\mathrm{PC}$ & .452 & .000 & .000 & .000 & .452 & .108 \\
\hline & S2T & .261 & 1.000 & 1.000 & 1.000 & .261 & .799 \\
\hline \multirow{2}{*}{$\begin{array}{l}\text { Q9. I feel confident } \\
\text { implementing SPM in my } \\
\text { teaching }\end{array}$} & $\mathrm{PC}$ & .188 & .275 & .146 & .079 & .188 & -.169 \\
\hline & S2T & .656 & .510 & .731 & .853 & .656 & .690 \\
\hline \multirow{2}{*}{$\begin{array}{l}\text { Q10. Feedback received } \\
\text { over the course was } \\
\text { valuable }\end{array}$} & $\mathrm{PC}$ & .378 & .488 & .488 & $.747^{*}$ & .378 & $.882^{* *}$ \\
\hline & S2T & .356 & .220 & .220 & .033 & .356 & .004 \\
\hline \multirow{2}{*}{$\begin{array}{l}\text { Q11. Provided opportunities } \\
\text { to engage with student } \\
\text { colleagues from other } \\
\text { disciplines }\end{array}$} & $\mathrm{PC}$ & .577 & . 149 & .149 & $.726^{*}$ & .577 & .518 \\
\hline & S2T & .134 & .725 & .725 & .041 & .134 & .188 \\
\hline \multirow{2}{*}{$\begin{array}{l}\text { Q12. Provided me with the } \\
\text { necessary tools to develop } \\
\text { and implement scenarios for } \\
\text { my course }\end{array}$} & PC & .407 & -.063 & -.231 & -.190 & .407 & -.044 \\
\hline & S2T & .317 & .882 & .582 & .652 & .317 & .918 \\
\hline
\end{tabular}

Note: $\mathrm{PC}=$ Pearson correlation. $\mathrm{S} 2 \mathrm{~T}=$ sig. $(2$-tailed). $\mathrm{N}=8$ in all instances. $*$ Correlation is significant at the 0.05 level (2-tailed). ** Correlation is significant at the 0.01 level (2-tailed).

Qualitative data was collated. In response to the question, "What would I tell a friend or colleague about this workshop?" participant responses included

"Phenomenal—excellent, practical, thoughtful design and content was evident from opening to close."

"It helps to clarify things in and out of education environment."

"I would tell them I found it effective."

"Awesome! Great to learn about SPM and use it as one of the alternative tools in pedagogy."
"It is informative and a great way to see the implementation/benefits of simulation in crossdisciplinary settings/environments. It was also great networking with faculty."

"Very informative and useful educational tool."

Participants also enumerated various applications for which this pedagogical tool could be utilized:

"In-unit staff development days."

"To underscore the importance of using 'best practice' tools/procedures in a health related physical fitness domain." 
Table 2b. Correlations from SPSS Analysis for SP methodology workshop 2017

\begin{tabular}{|c|c|c|c|c|c|c|c|}
\hline & \multicolumn{6}{|c|}{ Question number } \\
\hline & & Q7 & Q8 & Q9 & Q10 & Q11 & Q12 \\
\hline \multirow[t]{2}{*}{ Q1. Workshop Program } & $\mathrm{PC}$ & .000 & .452 & .188 & .378 & .577 & .407 \\
\hline & S2T & 1.000 & .261 & .656 & .356 & .134 & .317 \\
\hline \multirow[t]{2}{*}{ Q2. Material Provided } & $\mathrm{PC}$ & .188 & .000 & .275 & .488 & .149 & -.063 \\
\hline & S2T & .656 & 1.000 & .510 & .220 & .725 & .882 \\
\hline \multirow{2}{*}{$\begin{array}{l}\text { Q3. Balance between } \\
\text { presentations, discussions } \\
\text { and activities }\end{array}$} & $\mathrm{PC}$ & .188 & .000 & .146 & .488 & .149 & -.231 \\
\hline & S2T & .656 & 1.000 & .731 & .220 & .725 & .582 \\
\hline \multirow{2}{*}{$\begin{array}{l}\text { Q4. Time distribution of the } \\
\text { workshop }\end{array}$} & $\mathrm{PC}$ & -.044 & .000 & .079 & $.747^{*}$ & $.726^{*}$ & -.190 \\
\hline & S2T & .918 & 1.000 & .853 & .033 & .041 & .652 \\
\hline \multirow{2}{*}{$\begin{array}{l}\text { Q5. Organization of } \\
\text { workshop }\end{array}$} & $\mathrm{PC}$ & .000 & .452 & .188 & .378 & .577 & .407 \\
\hline & S2T & 1.000 & .261 & .656 & .356 & .134 & .317 \\
\hline \multirow{2}{*}{$\begin{array}{l}\text { Q6. Group/dyad work was } \\
\text { an effective medium during } \\
\text { the workshop }\end{array}$} & $\mathrm{PC}$ & -.218 & .108 & -.169 & $.882^{* *}$ & .518 & -.044 \\
\hline & S2T & .604 & .799 & .690 & .004 & .188 & .918 \\
\hline \multirow[b]{2}{*}{$\begin{array}{l}\text { Q7. The workshop taught } \\
\text { me to create roles for } \\
\text { simulation that meet } \\
\text { learning objectives for my } \\
\text { students }\end{array}$} & PC & 1 & $.731^{*}$ & $.957^{* *}$ & -.092 & -.280 & .691 \\
\hline & S2T & & .039 & .000 & .829 & .502 & .058 \\
\hline \multirow{2}{*}{$\begin{array}{l}\text { Q8. The workshop has } \\
\text { advanced my expertise in } \\
\text { planning, creating and } \\
\text { facilitating SPM }\end{array}$} & PC & $.731^{*}$ & 1 & $.717^{*}$ & .228 & .000 & $.932 *$ \\
\hline & S2T & .039 & & .045 & .587 & 1.000 & .001 \\
\hline \multirow{2}{*}{$\begin{array}{l}\text { Q9. I feel confident } \\
\text { implementing SPM in my } \\
\text { teaching }\end{array}$} & PC & $.957^{* * *}$ & $.717^{*}$ & 1 & -.118 & -.181 & $.708^{*}$ \\
\hline & S2T & .000 & .045 & & .780 & .668 & .049 \\
\hline \multirow{2}{*}{$\begin{array}{l}\text { Q10. Feedback received } \\
\text { over the course was } \\
\text { valuable }\end{array}$} & $\mathrm{PC}$ & -.092 & .228 & -.118 & 1 & .655 & -.031 \\
\hline & S2T & .829 & .587 & .780 & & .078 & .942 \\
\hline \multirow{2}{*}{$\begin{array}{l}\text { Q11. Provided opportunities } \\
\text { to engage with student } \\
\text { colleagues from other } \\
\text { disciplines }\end{array}$} & $\mathrm{PC}$ & -.280 & .000 & -.181 & .655 & 1 & -.235 \\
\hline & S2T & .502 & 1.000 & .668 & .078 & & .575 \\
\hline \multirow{2}{*}{$\begin{array}{l}\text { Q12. Provided me with the } \\
\text { necessary tools to develop } \\
\text { and implement scenarios for } \\
\text { my course }\end{array}$} & PC & .691 & $.932^{* *}$ & $.708^{*}$ & -.031 & -.235 & 1 \\
\hline & S2T & .058 & .001 & .049 & .942 & .575 & \\
\hline
\end{tabular}

Note: $\mathrm{PC}=$ Pearson correlation. $\mathrm{S} 2 \mathrm{~T}=$ sig. (2-tailed). $\mathrm{N}=8$ in all instances.

*. Correlation is significant at the 0.05 level (2-tailed). ** Correlation is significant at the 0.01 level (2-tailed);

"To use it as part of the workshops offered to international teaching assistants. In other words, to use it in educational/professional workshops to teaching assistants."

These participants' statements contribute depth and insight to the statistically significant data points analyzed from the quantitative surveys.

\section{Application of SP methodology within a teaching-learning context}

SP methodology can enhance student engagement. Our findings indicate that this workshop protocol supports SP methodology by reinforcing its application to learning objectives, developing a detailed scenario, and effective use debriefing. 


\subsection{Grounding simulation in learning objectives}

Participants expressed increased awareness of the importance of grounding simulation in clear, purposeful learning objectives. One participant noted, "You must be just as clear on what [students] are needing to learn and this frames how you teach them." Another commented, "It is imperative to understand that this notion of framing simulation based on learning objectives provides a clearer picture of how learning happens or comes together from the learner's lens." Simulation encounters that are closely developed and aligned with specific learning objectives were viewed as increasing the clarity and efficacy of the experience.

\subsection{Detailed scenario development}

In line with close adherence to learning objectives, participants expressed a heightened awareness of the depth of detail involved in scenario development. The provided template was viewed as a helpful resource to guide scenario development. The opportunity to participate in simulation as learners and facilitators served to both inform scenario development and elicit feedback from various perspectives.

\subsection{Debriefing}

The structure provided for debriefing differed from traditional feedback and was new for some participants. The response to this approach was largely positive; debriefing, with its focus on observed behaviours rather than on the individual, was viewed as valuable to the simulation experience. The structure was described by participants as "very effective," "clean and simple," and "extremely useful." Participants in the first workshop also noted that a demonstration would have been beneficial to introduce facilitation of the debriefing process prior to engaging in the activity; these demonstrations were embedded within the second workshop.

\section{Discussion}

The workshop offered insight into experientialeducation innovation. Although results of the study indicate that knowledge improves after participation in educational workshops, there is limited evidence regarding a similar improvement in skills. The workshop resulted in an increase in participants' knowledge about the application of SP methodology in the teaching-learning context, particularly in the classroom. Given the lack of studies conducted in comparable populations, further research is needed to determine how the application of educational workshops can enhance and sustain teaching skills. Further research is also needed to explore the use of this version of the workshop with faculty teaching other disciplines and among different learner cohorts.

Feedback from participants expressed an interest in maintaining or increasing the level of interactivity and experiential activities. Participants' interest in and enthusiasm for the workshop are apparent in the following qualitative comments:

"I would like to see how we could implement this at some 'less' practical programs, in LAPs [liberal arts programs] for example . . . and how to bridge it with hands-on programs in the humanities"

"Phenomenal, excellent, practical, thoughtful design captured my attention from opening to close"

"Difference between SPM and role play was very clear ... planning, organizing, and implementing a scenario using the template well designed"

One SP observed,

"It was a great opportunity to work with faculty and learn from other disciplines"

Additionally, participants offered suggestions for future workshops. Some suggested that workshops should offers videos to complement the process, while others recommended offering it to TAs and graduate students and using it within other experiential education and professional workshops.

The next step in this project will be to embed the SP methodology in the classroom and conduct focusgroup interviews with three groups of participants: faculty, simulators, and learners. This next step is underway and analysis of the data gathered from focus groups is pending.

The SP methodology workshop in 2017 maintained the positive components of the pilot workshop, but refinements were implemented in response to reflection and feedback received from the pilot. This workshop saw an increase in participant enrolment and diversification of faculty and staff in attendance. New simulated persons expressed interest in the project and were trained along with previously trained SPs who elected to continue with the program.

\subsection{Future considerations}

As budget and funding constraints pervade the healthcare and education sectors, it is important to explore the cost effectiveness of proposed investments. Historically, the costs associated with simulation have been high, which challenged the acknowledgement of its long-term benefits [16]; it should be noted, however, that these costs can vary from high to low, "depending on 
methods, technology, and fidelity of the simulation" [17] (p. 2). Cost effectiveness, therefore, is crucial if this educational technique is to be seen as a viable option for institutions [18].

There is a noticeable gap in the literature regarding cost effectiveness or evaluations of the investment return of simulation education [17] [18]. Individual organizations often attempt to decrease costs of simulation through anecdotal approaches or by brainstorming less expensive means to meet their particular education needs [19]. Peers or colleagues, for example, can be used as simulators, which can decease costs associated with hiring professional actors [17]. There is a push for simulation education to embed economic evaluations into the processes surrounding simulation projects [17]. One form of economic evaluation is a cost-effectiveness analysis that provides data "to assist decision-makers on how to allocate scarce resources effectively" [18] (p. 221). These components are scarce in simulation education as "economic evaluations are largely based in a quantitative research paradigm, whereas medical education and educational research is ... predominantly focused on qualitative research methods" [17] (p. 4). Thus, there is a large gap in the body of evidence to demonstrate the efficiency of simulated-learning approaches [17].

\section{Conclusion}

We hypothesize that educating educators to work and teach with SP methodology will provide opportunities for fostering student success, thus creating further opportunities for curriculum synergies. One such opportunity has been to use the experience and learning from this initiative to create a practicum course for theatre students, which will serve to provide simulators to educators across the university who have taken the simulation workshop. Embedding this methodology reinforces the importance of engaging students through scenarios that simulate the realities and complexities of practice - realities that often do not match the textbook portrayal—and, thus, contribute to students' success in their transition to the workplace milieu.

In general, the workshop was successful and laid a foundation for future training programs of this style. This foundation formed an effective outline for subsequent iterations of this workshop, most recently in June 2017, with plans for future workshops in discussion. Identified problems will allow us to further refine the workshop to meet the needs of educators looking to embed SP methodology in their curricula. Further, knowledge gained from the workshop will allow us to design a blueprint for a cross-disciplinary program to provide educators with training in simulation methodology and undergraduate students with an opportunity to practice and develop competencies in their respective fields. Future planning is underway to further develop the complexity and scope of this ambitious project.

\section{Acknowledgement}

The York University Academic Innovation Fund Grant Category I.

\section{References}

[1] Bosse, H. M., Nickel, M., Huwendiek, S., Schultz, J. H., \& Nikendei, C. (2015). Cost-effectiveness of peer role play and standardized patients in undergraduate communication training. BMC Medical Education, 15, 183. doi: 10.1186/s12909-015-0468-1.

[2] McCaughey, C., \& Traynor, M. (2010). The role of simulation in nurse education. Nurse Education Today, 30, 827-832. doi: 10.1016/j.nedt.2010.03.005

[3] Waxman, K. (2010). The development of evidence-based clinical simulation scenarios: guidelines for nurse educators. Journal of Nursing Education, 49, 29-35. doi: 10.3928/01484834-20090916-07

[4] Harder, B. N. (2010). Use of simulation in teaching and learning in health sciences: A systematic review. Journal of Nursing Education, 49, 23-28. doi: 10.3928/0148483420090828-08

[5] McGaghie, W., Issenberg, S., Petrusa, E., \& Scalese, R. (2010). A critical review of simulation-based medical education research: 2003-2009. Medical Education, 44, 50 63. doi: 10.1111/j.1365-2923.2009.03547.x

[6] McGaghie, W., Siddall, V., Mazmanian, P., \& Myers, J. (2009). Lessons for continuing medical education from simulation research in undergraduate and graduate medical education: Effectiveness of continuing medical education: American College of Chest Physicians Evidence-Based Educational Guidelines. Chest, 62S-68S. doi: $10.1378 /$ chest.08-2521

[7] Robertson, J. \& Bandali, K. (2008). Bridging the gap: Enhancing interprofessional education using simulation. Journal of Interprofessional Care, 22, 499-508. doi: $10.1080 / 13561820802303656$

[8] Bland, A. J., Topping, A., \& Wood, B. (2011). A concept analysis of simulation as a learning strategy in the education of undergraduate nursing students. Nurse Education Today, 31, 664-670. doi:10.1016/j.nedt.2010.10.013

[9] Asal, V., \& Blake, E. L. (2006). Creating simulations for political science education. Journal of Political Science Education, 2(1), 1-18. doi: 10.1080/15512160500484119 
[10] Kelly, M. A., Berragan, E., Husebø, S. E., \& Orr, F. (2016). Simulation in nursing education-international perspectives and contemporary scope of practice. Journal of Nursing Scholarship, 48, 312-321. doi: 10.1111/jnu.12208

[11] Taylor, C., Mills, A., Schmied, V., Dahlen, H., Shuiringa, W., \& Hudson, M. E. (2012). What works to engage young parents into services? Findings from an appreciative inquiry workshop. Contemporary Nurse: A Journal For The Australian Nursing Profession, 42, 258-271. doi:10.5172/conu.2012.42.2.258

[12] Dematteo, D., \& Reeves, S. (2011). A critical examination of the role of appreciative inquiry within an interprofessional education initiative. Journal of Interprofessional Care, 25, 203-208. doi: $10.3109 / 13561820.2010 .504312$

[13] Tosati, S., Lawthong, N., \& Suwanmonkha, S. (2015). Development of an appreciative inquiry and assessment processes for students' self-knowing and self-development. Procedia-Social and Behavioral Sciences, 191, 753-758. doi: 10.1016/j.sbspro.2015.04.422

[14] Watkins, S., Dewar, B., \& Kennedy, C. (2016). Appreciative inquiry as an intervention to change nursing practice in in-patient settings: An integrative review. International Journal Of Nursing Studies, 60, 179-190. doi: 10.1016/j.ijnurstu.2016.04.017

[15] Rudolph, L. W., Raemer, D. B., \& Simon, R. (2014). Establishing a safe container for learning in simulation: The role of the presimulation briefing. Simulation in Healthcare. 9, 339-349. doi: 10.1097/SIH.0000000000000047

[16] Lateef, F. (2010). Simulation-based learning: Just like the real thing. Journal of Emergencies, Trauma, and Shock, 3, 348-352. doi: 10.4103/0974-2700.70743

[17] Maloney, S., \& Haines, T. (2016). Issues of cost-benefit and cost-effectiveness for simulation in health professions education. Advances in Simulation, 1:13. doi: 10.1186/s41077-016-0020-3

[18] Isaranuwatchai, W., Brydges, R., Carnahan, H., Backstein, D., \& Dubrowski, A. (2014). Comparing the costeffectiveness of simulation modalities: A case study of peripheral intravenous catheterization training. Advances in Health Sciences Education, 19, 219-232. doi: 10.1007/s10459-013-9464-6

[19] Chichester, M., Hall, N. J., Wyatt, T. L., \& Pomilla, R. (2014). A cost-effective approach to simulation-based team training in obstetrics. Nursing for Women's Health, 18, 500507. doi: 10.1111/1751-486X.12162 